\title{
Asymptotic Effective Piezoelectric Coefficient Solution of Piezoresponse Force Microscopy for a Transversely Isotropic Piezoelectric Film
}

\author{
J. H. Wang ${ }^{1, a}$ \\ ${ }^{1}$ School of Mechanics, Civil Engineering and Architecture, Northwestern Polytechnical University, Xi'an Shaanxi, 710129, People's \\ Republic of China
}

\begin{abstract}
Based on the coupled theory, a simple explicit solution of piezoresponse force microscopy (PFM) in determining the effective piezoelectric coefficient for an ultra-thin transversely isotropic piezoelectric film bonded to a rigid conducting substrate is obtained, using the Taylor expansion and homogeneous assumption. And it is found to be exactly the same as the well-known result for the case of piezoelectric thin film clamped between flat rigid electrodes for homogeneous external electric field. The electric charge and the distance from the image charge model are also derived and the influences of the film thickness and substrate permittivity on the effective piezoelectric coefficient are then discussed. The obtained results can be used to quantitatively interpret the PFM signals and directly detect the piezoelectric constant through PFM for an ultra-thin film or supply important information for constructing a reliable formula to describe the thickness effect.
\end{abstract}

\section{Introduction}

Making use of the converse piezoelectric effect, piezoresponse force microscopy (PFM), has emerged as a widely used technique for investigating ferroelectric and piezoelectric samples to characterize material properties at the nanometer scale [1-2]. Application of PFM to measure the two-dimensional domain structures and switching behavior in ferroelectrics and quantitatively detect the piezoelectric coefficients of piezoelectric films necessitates the development of reliable analytical methods to describe the involved image formation mechanisms [2].

Essentially, theoretical investigation of PFM is a boundary value problem in piezoelectric solids. For a point electric charge on top of a transversely isotropic half plane Karapetian et al. [3] presented a piezoelectric Green's function solution to calculate the corresponding surface displacements. Kalinin and Bonnell [4] and Kalinin et al. [5] adopted the analytical solutions for indentation problems to interpret the PFM responses. Instead of solving the piezoelectric equations, an alternative decoupled method, which is based on the assumption that the electric field under which the surface deformation is induced is purely calculated for the rigid dielectric, was originally proposed by Ganpule et al. [6] to account for the effect of $90^{\circ}$ domain walls on PFM image. Agronin et al. [7] adapted a one-dimensional form to obtain piezoelectric strain coefficient $d_{33}$ in ferroelectrics while Felten et al. [8] extended the decoupled approach to threedimensional cases. The decoupled approach neglects the electromechanical couplings and treats the piezoelectric strain as an eigenstrain. By doing that, the analysis becomes rather simple and is widely used to investigate PFM responses [9-13].

However, due to the neglect of the electromechanical coupling, the accuracy of the decoupled method is ambiguous and it is desirable to develop a coupled theory to rigorously calculated PFM responses [9]. Wang et al. [14] extended the indentation analysis for piezoelectric films [15] to PFM problems and presented a closed-form solution for the effective piezoelectric coefficient. Following the same method, Pan et al.16 conducted a more detailed coupled analysis for the PFM signals of a transversely isotropic piezoelectric half space. The obtained results for the effective piezoelectric coefficient from the coupled theory for half space are implicit and complicated combinations of material constants and thus very difficult to use to detect piezoelectric constants directly.

Except the work of Morozovska et al. [11] extending the decoupled theory to surface layers and thin films, most of the aforementioned theoretical investigations are based on the half space assumption,

\footnotetext{
${ }^{a}$ Corresponding author: wangjh@nwpu.edu.cn
} 
without considering the effect of the film thickness. In this contribution, we investigate the PFM problem of an ultra-thin transversely isotropic piezoelectric film with the coupled theory presented in Refs. [14-16]. Using the Taylor expansion and homogeneous assumption, we obtain simple explicit solutions for the effective piezoelectric coefficient and the corresponding electric charge and distance from the image charge model. The influences of the film thickness and substrate permittivity on the effective piezoelectric coefficient are analyzed.

\section{Fundamental Equations}

A transversely isotropic piezoelectric film is perfectly bonded to a rigid conducting substrate as shown in Figure 1, above which a conducting axisymmetric SPM tip with an input potential $\phi_{0}$ is located. The cylindrical polar coordinate system $(r, \theta, z)$ is introduced and the piezoelectric film is isotopic in the $(r, \theta)$ plane. The thickness of the film is denoted by " $t$ " and the tip curvature radius " $R_{0}$ ". Using the simple image charge model, the input potential $\phi_{0}$ is regarded as an electric charge " $Q$ " at a distance " $d$ " above the surface.

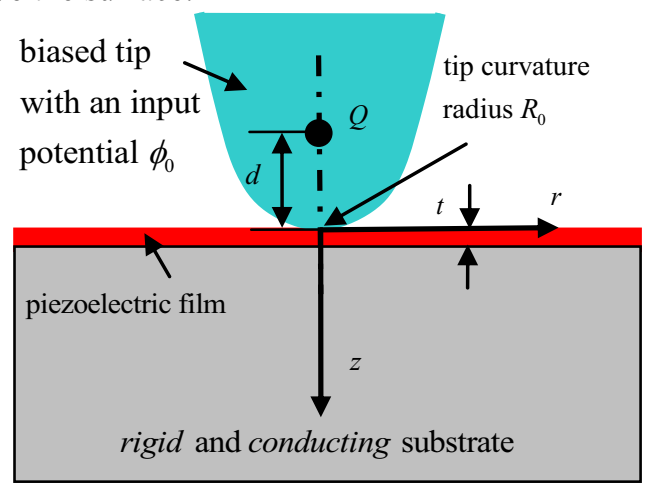

Figure 1. Schematic of a PFM Experiment Operated on a Piezoelectric Film with the Thickness $t$.

The input potential $\phi_{0}$ is approximated as an electric charge $Q$ at the distance $d$ above the surface.

For a transversely isotropic piezoelectric film, the constitutive equation is:

$$
\left\{\begin{array}{l}
\sigma_{r r} \\
\sigma_{\theta \theta} \\
\sigma_{z z} \\
\sigma_{r z} \\
D_{r} \\
D_{z}
\end{array}\right\}=\left(\begin{array}{cccccc}
c_{11} & c_{12} & c_{13} & 0 & 0 & e_{31} \\
c_{12} & c_{11} & c_{13} & 0 & 0 & e_{31} \\
c_{13} & c_{13} & c_{33} & 0 & 0 & e_{33} \\
0 & 0 & 0 & c_{44} & e_{15} & 0 \\
0 & 0 & 0 & e_{15} & -\epsilon_{11} & 0 \\
e_{31} & e_{31} & e_{33} & 0 & 0 & -\epsilon_{33}
\end{array}\right\}\left\{\begin{array}{c}
\varepsilon_{r r} \\
\varepsilon_{\theta \theta} \\
\varepsilon_{z z} \\
2 \varepsilon_{r z} \\
-E_{r} \\
-E_{z}
\end{array}\right\}
$$

where $\sigma_{i j}$ and $D_{k}$ are the stress and electric displacement components, respectively, $c_{i j}, e_{i j}$ and $\epsilon_{i j}$ are the elastic, piezoelectric and dielectric constants, respectively. The infinitesimal strain $\varepsilon_{i j}$ and the electric field $E_{k}$ are related to the displacement components $u_{r}, u_{z}$ and the electric potential $\phi$ by:

$$
\begin{aligned}
& \varepsilon_{r r}=\frac{\partial u_{r}}{\partial r}, \varepsilon_{\theta \theta}=\frac{u_{r}}{r}, \varepsilon_{z z}=\frac{\partial u_{z}}{\partial z}, 2 \varepsilon_{r z}=\frac{\partial u_{r}}{\partial z}+\frac{\partial u_{z}}{\partial r} \\
& E_{r}=-\frac{\partial \phi}{\partial r}, E_{z}=-\frac{\partial \phi}{\partial z}
\end{aligned}
$$

Without considering the contact force between the biased tip and the film upper-surface, we have the stress free conditions $\sigma_{r z}(r, 0)=0, \sigma_{z z}(r, 0)=0$. The corresponding boundary conditions for the rigid and conducting substrate are $u_{r}(r, t)=0, u_{z}(r, t)=0$ and $\phi(r, t)=0$. Following the same method presented in Ref. [14], the surface potential $(z=0)$ and displacement along the $z$ axis could be expressed as:

$$
\begin{aligned}
& \phi(r, 0)=\frac{Q}{2 \pi} \int_{0}^{\infty} \frac{B_{2}(\xi) \mathrm{e}^{-\xi d}}{\left[\epsilon_{e} B_{2}(\xi)+B_{3}(\xi)\right]} J_{0}(\xi r) \mathrm{d} \xi \\
& u_{z}(r, 0)=\frac{Q}{2 \pi} \int_{0}^{\infty} \frac{B_{1}(\xi) \mathrm{e}^{-\xi d}}{\left[\epsilon_{e} B_{2}(\xi)+B_{3}(\xi)\right]} J_{0}(\xi r) \mathrm{d} \xi \\
& D_{z}(r, 0)=\frac{Q}{2 \pi} \int_{0}^{\infty} \frac{B_{3}(\xi) \xi \mathrm{e}^{-\xi d}}{\left[\epsilon_{e} B_{2}(\xi)+B_{3}(\xi)\right]} J_{0}(\xi r) \mathrm{d} \xi
\end{aligned}
$$

where $B_{i}(\xi \tilde{t})(i=1,2,3)$ are functions of the film thickness $t$ and material properties. In Eq. (3), $\xi$ is the Hankel transformation space coordinate, $\epsilon_{e}$ the ambient dielectric property and $\tilde{t}$ denotes $t / d$. And $J_{0}(\xi r)$ is the 0th order Bessel function.

Then the effective piezoelectric coefficient $d_{33}^{\text {eff }}$ $\left(=u_{z}(0,0) / \phi(0,0)\right)$ for a thin film on a rigid and conducting substrate is expressed as follows:

$$
d_{33}^{\text {eff }}=\frac{\int_{0}^{\infty} \frac{B_{1}(\xi \tilde{t})}{\left[\epsilon_{e} B_{2}(\xi \tilde{t})+B_{3}(\xi \tilde{t}) e^{-\xi} d \xi\right.}}{\int_{0}^{\infty} \frac{B_{2}(\xi \tilde{t})}{\left[\epsilon_{e} B_{2}(\xi \tilde{t})+B_{3}(\xi \tilde{t}) e^{-\xi} d \xi\right.}}
$$

In Eq. (4), $B_{i}(\xi \tilde{t})(i=1,2,3)$ are unknown functions which should be determined from boundary conditions. In the image charge model, the input potential is viewed as an equivalent electrical charge. So it requires that (1) the potential at the tip apex equals the voltage applied on the conductive SPM tip; and (2) the radius of curvature of the equipotential surface at tip apex equals the radius of curvature of SPM tip[16], from which the effective electrical charge " $Q$ " and distance " $d$ are determined: 


$$
\begin{aligned}
& \frac{Q}{2 \pi d} \int_{0}^{\infty} \frac{B_{2}(\xi \tilde{t})}{\left[\epsilon_{e} B_{2}(\xi \tilde{t})+B_{3}(\xi \tilde{t})\right]} e^{-\xi} d \xi=\phi(0,0) \\
& \frac{Q}{2 \pi d^{2}} \int_{0}^{\infty} \frac{B_{3}(\xi \tilde{t})}{\left[\epsilon_{e} B_{2}(\xi \tilde{t})+B_{3}(\xi \tilde{t})\right]} e^{-\xi} \xi d \xi=D_{z}(0,0) \\
& d \mid \frac{\int_{0}^{\infty} \frac{B_{3}(\xi \tilde{t})}{\left[\epsilon_{e} B_{2}(\xi \tilde{t})+B_{3}(\xi \tilde{t})\right]} e^{-\xi} \xi d \xi}{\int_{0}^{\infty} \frac{\epsilon_{e} B_{2}(\xi \tilde{t})}{\left[\epsilon_{e} B_{2}(\xi \tilde{t})+B_{3}(\xi \tilde{t})\right]} e^{-\xi} \frac{\xi^{2}}{2} d \xi \mid=R_{0}}
\end{aligned}
$$

\section{Asymptotic solutions}

Generally speaking, it is almost impossible to solve Eqs. (4-5) analytically. However, considering the film as "ultra-thin", that is $t / d \rightarrow 0$, we can expand the integral expressions in Taylor series at $\tilde{t}=0$ and keep the first non-zero terms in Eqs. (4-5). Accordingly, Eqs. (4-5) transfer to a very simple form and the asymptotic solutions for the effective piezoelectric coefficient, electric charge, and distance of the ultra thin piezoelectric film are derived as:

$$
\begin{aligned}
& d_{33}^{e f f}=C_{1} \\
& Q=6 \pi \in_{e} R_{0} \phi(0,0) \\
& d=\sqrt{3 \in_{e} C_{2} t R_{0}} \\
& D_{z}(0,0)=\phi(0,0) /\left(C_{2} t\right)
\end{aligned}
$$

where $C_{1}$ and $C_{2}$ are constants related to material properties. Similar to the half space results [15-16], it is difficult to directly detect piezoelectric coefficients through PFM without the knowledge of the relationships between $C_{1}$ and $C_{2}$ and the material constants. Note that the electric field of the PFM tip progressively becomes homogeneous with the film thickness decreasing. Thus it is reasonable to assume that the strain and the electric field are uniform throughout the film thickness for an ultra-thin film [17], resulting in:

$$
\begin{aligned}
& \varepsilon_{z z}=\frac{\partial u_{z}}{\partial z}=\frac{u_{z}(r, t)-u_{z}(r, 0)}{t}, \\
& E_{z}=-\frac{\partial \phi}{\partial z}=\frac{\phi(r, 0)-\phi(r, t)}{t}
\end{aligned}
$$

Meanwhile, assuming the radial displacement component $u_{r}$ independent of $z$ and considering the rigid substrate boundary condition $u_{r}(r, t)=0$, we have:

$$
u_{r}=0, \varepsilon_{r r}=\varepsilon_{\theta \theta}=0 \quad(0 \leq r<\infty, 0<z<t)
$$

ubstituting Eqs. (8) and (9) into Eq. (1), the following relationship between the surface displacement $u_{z}(r, 0)$ and surface potential $\phi(r, 0)$ is derived:

$$
\begin{aligned}
& c_{33} u_{z}(r, 0)+e_{33} \phi(r, 0)=0 \\
& -e_{33} u_{z}(r, 0)+\epsilon_{33} \phi(r, 0)=D_{z}(r, 0) t
\end{aligned}
$$

According to the definition of the effective piezoelectric coefficient, one can get from Eq. (10) that:

$$
d_{33}^{e f f}=-u_{z}(0,0) / \phi(0,0)=e_{33} / c_{33}
$$

and

$$
D_{z}(r, 0)=\left(e_{33}^{2} / c_{33}+\epsilon_{33}\right) \phi(r, 0) / t
$$

By comparisons between Eqs.(11-12) and Eqs.(67), we obtain the relationships:

$$
C_{1}=e_{33} / c_{33}, C_{2}=1 /\left(e_{33}^{2} / c_{33}+\epsilon_{33}\right)
$$

In other way, one can numerically calculated the effective piezoelectric coefficient $d_{33}^{\text {eff }}$ and a normalized expression $d^{2} /\left(3 \in_{e} R_{0} t\right)$ from Eqs.(4-5) and compared them with $e_{33} / c_{33}$ and $1 /\left(e_{33}^{2} / c_{33}+\epsilon_{33}\right)$ respectively as shown in Figure 2 . It is clear that as $\tilde{t}$ varies from 0.5 to 0.01 , the calculated $d_{33}^{\text {eff }}$ from Eq.(4) do approach the results given in Eq.(11) and $d^{2} /\left(3 \in_{e} R_{0} t\right)$ equals $1 /\left(e_{33}^{2} / c_{33}+\epsilon_{33}\right) \quad$ verifying the relationships in Eq.(13) .

Actually, taking advantage of the relationship between material constants $d_{33}=e_{33} / c_{33}-2 c_{13} / c_{33} d_{31}$, Eq. (11) can be rewritten as:

$$
d_{33}=d_{33}^{e f f}-2 c_{13} / c_{33} d_{31}
$$

This is the expression between the effective piezoelectric coefficient from PFM and the true one for an ultra-thin piezoelectric film. To be more specific, if we introduce the relationship between the compliance and the stiffness constants $c_{13} / c_{33}=s_{13} /\left(s_{11}+s_{12}\right)$,Eq.(14) is exactly the same as the well-known piezoelectric coefficient result for the case of piezoelectric thin film clamped between flat rigid electrodes for homogeneous external electric field [18], and the effective piezoelectric coefficient from the decoupled theory for a piezoelectric film on a rigid and conducting substrate at $t / d \rightarrow 0$ reconsidering the transverse isotropy of the film [11]. This illustrates that the point-contact-like PFM geometry transits to the flat capacitor response for films rather thinner than the tip size. 
(a)

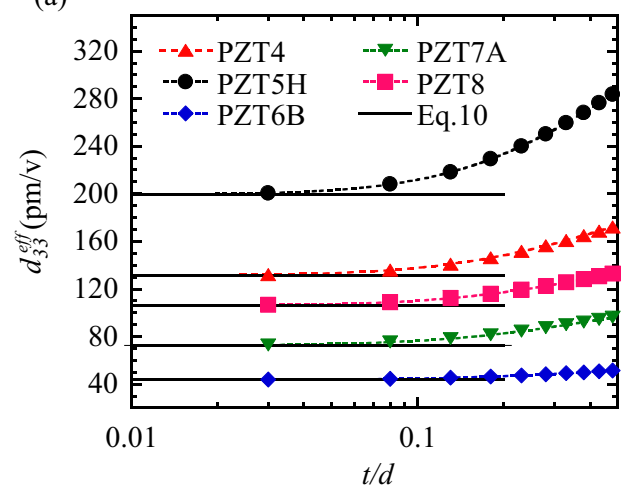

(b)

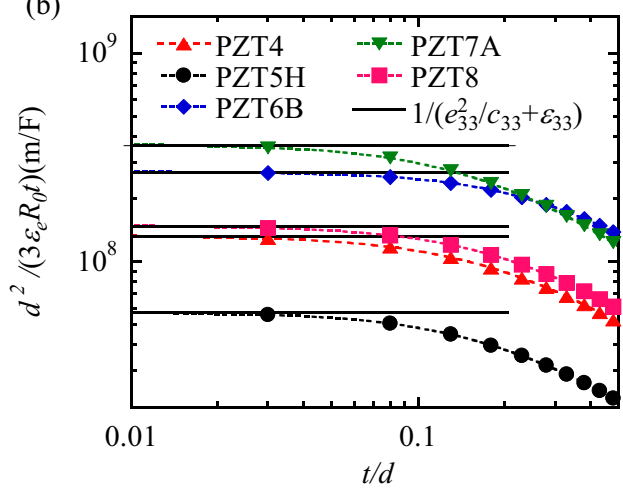

Figure 2. (a) Effective Piezoelectric Coefficient $d_{33}^{\text {eff }}$; (b) $d^{2} /\left(3 \in_{e} R_{0} t\right)$ as the Normalized Thickness $t / d$ Varies from 0.01 to 0.5 .

The obtained asymptotic solution for the effective piezoelectric coefficient, which is only a division of the piezoelectric coefficient $e_{33}$ by the elastic constant $c_{33}$ or a simple and explicit relationship with true piezoelectric coefficient $d_{33}$ and other material constants, and the electric charge and distance in the image charge model can be directly used to detect the piezoelectric coefficient with PFM if the film could be deemed as ultra-thin. That is $t / d<0.03$ from Figure 2 at which the discrepancies between the calculated values and those from Eq.(14) are less than 1\%. Taking the piezoelectric material PZT-4 for example, we have $R / t>3.18 \times 10^{5}$ from Eq. (7). This large value represents that the biased tip is much blunt and it is similar to a flat-ended punch.

It is already known that the calculated effective piezoelectric coefficient from the half space solutions is much lower than the true coefficient $d_{33}$ [15-16]. From Figure 2, it is demonstrated that as the normalized thickness decreases, the effective piezoelectric coefficient $d_{33}^{\text {eff }}$ declines to the ultra-thin film solution. For PZT-4, $d_{33}=289.4(\mathrm{pm} / \mathrm{V})$ while $d_{33}^{\text {eff }}$ for half space is $250(\mathrm{pm} / \mathrm{V})$ and for ultra-thin film $131.3(\mathrm{pm} / \mathrm{V})$ (Figure 3).It means that the effective piezoelectric coefficient from PFM is lower than the true piezoelectric coefficient and the film thickness has an obviously large influence on it. In case of a sharp point SPM tip when the film can not be seen as an ultra-thin one or a half space, thickness effect must be carefully investigated. Then the obtained asymptotic solutions as $t / d \rightarrow 0$ would supply important information for constructing a reliable formula to describe the thickness effect.

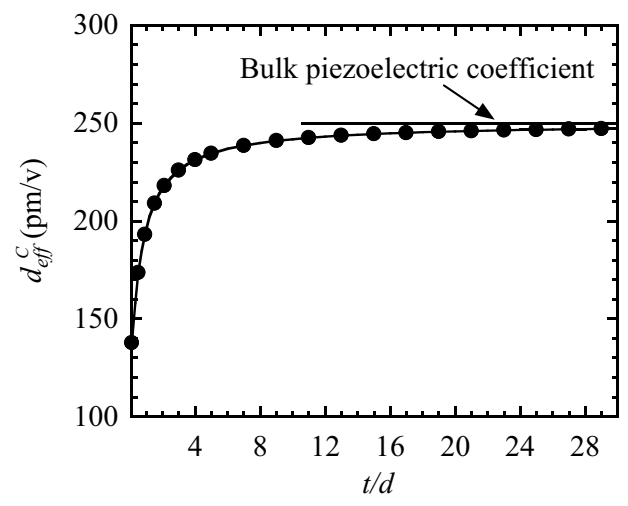

Figure 3. The Effective Piezoelectric Coefficient $d_{33}^{\text {eff }}$ versus the Normalized Thickness $t / d$.

Actually if the substrate is assumed to be insulating, e.g. the substrate permittivity approach infinity, the corresponding boundary condition becomes $D_{z}(r, t)=0$. From Eq.(9), we can obtain that the electric potential within the ultra-thin film is constant and so generates no surface displacement and gives rise to a zero $d_{33}^{\text {eff }}$. A similar conclusion could be found in Ref. [11]. Thus a metallic substrate or with a giant permittivity is recommended to conduct a PFM experiment on thin film to explore the piezoelectric coefficient in order to suppress the thickness dependence.

\section{Conclusions}

In summary, using the Taylor expansion and homogeneous assumption, unlike the complicated implicit combination of material constants for a piezoelectric half space, a simple explicit solution for the effective piezoelectric coefficient of an ultra-thin piezoelectric film bonded to a rigid and conducting substrate is obtained. The effective electric charge and the distance in the image charge model are also derived. The asymptotic solutions is exactly the same as the well-known result for the case of piezoelectric thin film clamped between flat rigid electrodes for homogeneous external electric field, and the decoupled effective piezoelectric coefficient solution for a piezoelectric film on a rigid and conducting substrate at reconsidering the transverse isotropy of the film. The influences of the film thickness on the effective piezoelectric coefficient are discussed. A metallic substrate or with a giant permittivity is recommended to conduct a PFM experiment on thin film to explore the piezoelectric coefficient to suppress the thickness dependence. The obtained results can be directly used to detect the piezoelectric constant through PFM for an 
ultra-thin film or supply important information for constructing a reliable formula to describe the thickness effect.

\section{Acknowledgments}

The authors are grateful for the financial support of this work by the National Science Foundation of China (NO.11502206) and the Fundamental Research Foundation of Northwestern Polytechnical University (No. JCY20130124).

\section{References}

1. M. Alexe, A. Gruverman, Nanoscale Characterization of Ferroelectric Materials (Springer, New York, 2004).

2. J. Tobias, H. Ákos, S. Elisabeth, Appl. Phys. Lett., 91, 25 (2007).

3. E. Karapetian, I. Sevostianov, K. Kachanov, Philos. Mag. B, 80, 3 (2000).

4. S.V. Kalinin, D.A. Bonnell, Phys. Rev. B, 65, 6 (2002).

5. S.V. Kalinin, E. Karapetian, M. Kachanov, Phys. Rev. B, 70, 18 (2004).

6. C. Ganpule, S.V. Nagarjan, H. Li, A.S. Ogale, D.E. Steinhauer, S. Aggarwal, E. Williams, R. Ramesh, D.P. Wolf, Appl. Phys. Lett., 77,2 (2000).

7. A. Agronin, M. Molotskii, Y. Rosenwaks, E. Strassburg, A. Boag, S. Mutchnik, G. Rosenman, J. Appl. Phys., 97, 8 (2005).

8. F. Felten, G.A Schneider, J.M. Saldan, and S.V. Kalinin, J. Appl. Phys., 96, 1(2004).

9. S.V. Kalinin, E.A. Eliseev, A.N. Morozovska, Appl. Phys. Lett., 88, 23 (2006).

10. E.A. Eliseev, S.V. Kalinin, S. Jesse, S.L. Bravina, A.N. Morozovska, Appl. Phys. Lett., 102, 1 (2007).

11. A.N. Morozovska, E.A. Eliseev, S.V. Kalinin, J. Appl. Phys., 102, 7 (2007).

12. D. Scrymgeour, V. Gopalan, Phys. Rev. B, 72, 2 (2005).

13. L.L. Tian, A. Vasudevarao, A. Morozovska, N. Eliseev, E., S.V. Kalinin, V. Gopalan, J. Appl. Phys. ,104, 7 (2008).

14. J.H. Wang, C.Q.Chen, T.J. Lu, J. Mech. Phys. Solids, 56, 56 (2008).

15. J.H. Wang, C.Q. Chen, Appl. Phys. Lett., 99, 17 (2011).

16. K.Pan, Y.Y.Liu, S.H.Xie, Y.M.Liu, J.Y.Li, Acta Mater., 61, 18 (2013).

17. X.G.Ning, M.Lovell, W.S.Slaughter, Wear, 26, 7 (2006).

18. K.Lefki, G. J. M Dormans., J. Appl. Phys. 76 (1994). 